\title{
Regards croisés sur les paysages du maïs au Mexique
}

Stratégies paysannes de gestion de la biodiversité cultivée

Marianna Fenzi, Maria Gabriela Zurita-Benavides et Jorge Quetzal Argueta Prado

\section{CpenEdition}

\section{Journals}

Édition électronique

URL : https://journals.openedition.org/ethnoecologie/7159

DOI : 10.4000/ethnoecologie.7159

ISSN : 2267-2419

Éditeur

Laboratoire Éco-anthropologie

Référence électronique

Marianna Fenzi, Maria Gabriela Zurita-Benavides et Jorge Quetzal Argueta Prado, « Regards croisés sur les paysages du maïs au Mexique », Revue d'ethnoécologie [En ligne], Supplément 2 | 2021, mis en ligne le 25 novembre 2021, consulté le 20 décembre 2021. URL : http://journals.openedition.org/ ethnoecologie/7159; DOI : https://doi.org/10.4000/ethnoecologie.7159

Ce document a été généré automatiquement le 20 décembre 2021.

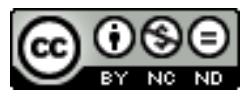

Revue d'ethnoécologie est mis à disposition selon les termes de la licence Creative Commons Attribution - Pas d'Utilisation Commerciale - Pas de Modification 4.0 International. 


\title{
Regards croisés sur les paysages du maïs au Mexique
}

\author{
Stratégies paysannes de gestion de la biodiversité cultivée \\ Marianna Fenzi, Maria Gabriela Zurita-Benavides et Jorge Quetzal \\ Argueta Prado
}

1 Dans ce numéro, nous présentons un riche éventail de textes sur la diversité du maïs au Mexique en relation avec les pratiques de l'agriculture paysanne. Les débats scientifiques sur ce thème s'inscrivent dans une réflexion sur la conservation de la biodiversité que la Revue d'ethnoécologie accueille et nourrit depuis longtemps. Les auteurs du présent dossier partagent donc un même objet de recherche, le maïs, qu'ils interrogent à des échelles différentes et avec une grande variété de points d'observation (expériences directes, analyses nationales, études locales) : pourquoi et pour qui conserver (Bahuchet et al. 2000, Rodríguez 2011, Swart et al. 2018) ? Comment étudier et mesurer un objet en constante évolution, tel que la biodiversité ? (Aguirre Salcedo \& Ceccon 2020, Nicholls et al. 2020, Iermanó et al. 2020) Comment analyser des pratiques et des phénomènes qui conservent mais, dans le même temps, transforment cette diversité (Dumez 2010, Cunha Ávila et al. 2017) ? La pertinence de certaines catégories d'analyse et les liens entre biodiversité et diversité culturelle (Virtanen 2019, Roué 2006) sont aussi au cœur de ces textes. Au Mexique, ces questionnements ont particulièrement suscité un intérêt dans le domaine de l'anthropologie et de l'écologie. Mais ils ont également été étudiés par des agronomes impliqués dans des programmes de recherche en faveur de l'agriculture paysanne.

Ce recueil de travaux a comme point de départ des rencontres organisées en 2014 : une journée internationale d'étude au Muséum national d'Histoire naturelle (MNHN) intitulée Regards croisés sur la biodiversité cultivée et deux journées en Aquitaine. À l'occasion de ces dernières, certains auteurs des textes ont été accueillis sur place par AgroBio Périgord, une association d'agriculteurs qui, depuis une vingtaine d'années, cultivent, sélectionnent et conservent des variétés hétérogènes de maïs. C'est en raison de l'apport précieux de cette association que nous avons voulu inclure leur témoignage dans ce recueil. Ces rencontres ont été possibles grâce au soutien de l'Action 
Thématique du Muséum (ATM) Savoirs naturalistes, expertise et politiques de la biodiversité, de la Red Patrimonio Biocultural de México, au Mexique ${ }^{1}$ et de l'association AgroBio Périgord. Le projet suisse Future Food Initiative nous a enfin soutenu dans la dernière phase d'édition et de traduction.

3 La réflexion autour de la distribution géographique, de la conservation et de la gestion à la ferme de la diversité des maïs mexicains constitue le fil rouge de ce numéro. La production scientifique sur cette espèce dans son centre d'origine, le Mexique, est assez abondante et a été caractérisée, ces deux dernières décennies, par de nombreuses interrogations et controverses, telles que celle sur l'érosion génétique du maïs (Dyer et al. 2014, Perales \& Golicher 2014, Brush et al. 2015). Loin de vouloir formuler des conclusions univoques autour de ces questions, nous revenons sur les débats les plus récents, au prisme de la place de l'agriculture paysanne et des variétés locales dans le contexte de l'agriculture mexicaine. Grâce à la variété des idées et des approches fournies dans ce numéro par des expériences locales et des analyses issues d'études à l'échelle nationale, nous espérons aider au développement de nouvelles pistes pour les sciences de la conservation au Mexique. En effet, le cas mexicain est singulièrement à la croisée de tous les enjeux sur la légitimité et les spécificités de conservation de la biodiversité cultivée par rapport à la biodiversité dite "sauvage », en particulier dans les arènes institutionnelles.

4 La Convention sur la diversité Biologique (CBD), signée à Rio en 1992, a grandement participé à formaliser une nouvelle gouvernance de la biodiversité, non plus seulement structurée autour des collections et des banques de gènes, mais sur l'identification et la promotion de pratiques in situ. Cette dernière approche est censée permettre l'évolution des populations en accord avec les changements environnementaux (Bennett 1968, Bretting \& Duvick 1997, Brush 1999). Ainsi, le fameux préambule de la convention précise : «les États sont responsables de la conservation de leur diversité biologique et de l'utilisation durable de leurs ressources biologiques [...] la conservation de la diversité biologique exige essentiellement la conservation in situ des écosystèmes $»^{2}$. Malgré les évolutions dans le domaine de la biologie de la conservation depuis la CBD, la biodiversité est encore souvent présentée par les institutions scientifiques et par les organisations internationales comme un stock de ressources figées menacées de disparition. Le but de la conservation est alors celui de tendre à l'exhaustivité des collections (sous forme d'objets physiques ou d'informations numériques) plutôt que d'intervenir sur les facteurs qui génèrent et rendent évolutive la biodiversité (Fenzi \& Bonneuil 2016). En outre, la conservation in situ reste marginalisée et critiquée comme " une distraction coûteuse et un gaspillage de fonds ", qui privent de ressources la conservation ex situ (Sadras 2018).

5 Outre cette vision qui oppose conservations in situ et ex situ (dans les banques de gènes), existe également l'idée que la biodiversité est liée aux savoirs sur la nature inscrits dans des activités agricoles perçues non plus seulement comme destructrices mais aussi comme productrices de diversité (Bretting \& Duvick 1997, Brush 1999, FAO 2019). Ces thématiques ont surtout suscité un intérêt au cours de ces dernières années et, après des débats intenses au sein des institutions internationales, en particulier de la FAO, une résolution sur les droits des agriculteurs a finalement été adoptée par le Conseil des droits de l'homme le 28 septembre 2018 : «Les paysans et les autres personnes travaillant dans les zones rurales sont titulaires du droit aux semences [...] qui englobe : [...] le droit de conserver, d'utiliser, d'échanger et de vendre des semences de ferme ou 
du matériel de multiplication [...]. Les États reconnaîtront aux paysans le droit d'utiliser leurs propres semences ou d'autres semences locales de leur choix, et de décider des cultures et espèces qu'ils souhaitent cultiver. Les États prendront des mesures appropriées pour appuyer les systèmes de semences paysannes et favoriseront l'utilisation des semences paysannes et l'agrobiodiversité " ${ }^{3}$.

6 L'élargissement, par certaines arènes scientifiques et institutionnelles, des contours de la gestion de la biodiversité pour prendre en compte d'autres éléments (culturels, alimentaires...) et de nouveaux acteurs (agriculteurs, communautés locales...) n'a pas encore entraîné dans la pratique de véritable renouvellement de la manière de concevoir la conservation et l'usage de la diversité cultivée. La plupart des projets portant sur les ressources génétiques des plantes visent essentiellement à stocker et à connaître la diversité des plantes cultivées par des pratiques de génotypage ${ }^{4}$. Ce travail d'extraction et d'archivage d'informations est sans doute très important mais, pour l'instant, ni les agriculteurs ni la conservation dynamique de ces ressources ne semblent en tirer des bénéfices. Il reste beaucoup à faire pour reconnaître la place centrale des agriculteurs dans la conservation et l'évolution de la diversité agricole. Celle-ci nécessiterait par ailleurs la traduction, dans les politiques publiques et lois nationales, de la résolution de 2018 sur les droits des agriculteurs.

Qu'est-ce qui freine donc la mise en valeur, non pas tant de la biodiversité en ellemême, mais de ce qui lui permet de perdurer : les pratiques agricoles, les dynamiques biologiques et culturelles qui la sous-tendent?

8 Les textes réunis ici identifient certaines tendances et transformations, sans se limiter à l'analyse de la distribution et au calcul de la diversité des variétés de maïs. Ils réservent une place centrale aux pratiques des agriculteurs et nous permettent d'aborder la biodiversité cultivée comme le résultat d'efforts mobilisés dans un constant effort d'adaptation. L'approche de la multiplicité des systèmes agricoles traditionnels et de leurs transformations est d'ailleurs illustrée dans ces textes par l'exemple de la «milpa», espace cultivé en maïs typique du système d'agriculture sur brûlis mexicain. La littérature scientifique et de vulgarisation décrit un système caractérisé par l'association de maïs, haricots et courges alors que la réalité nous offre un éventail de pratiques qui souvent ne rentrent pas dans ce schéma. Dans les milpas nous pouvons trouver des cultures commerciales destinées à la vente. Souvent, les milpas se présentent aussi sous la forme d'une monoculture de maïs vouée à l'autoconsommation, perçue comme un effort de rentabilisation par une paysannerie occupée à d'autres activités, y compris non-agricoles. Mais l'attention aux pratiques culturales ne doit pas se borner à l'observation du travail dans les champs. En nous intéressant à la diversité du maïs, nous allons découvrir que les frontières entre le maïs criollo, natif ou indigène, et les variétés modernes et hybrides sont rendues perméables par les pratiques des agriculteurs et la circulation constante des semences. C'est donc grâce à l'inventivité des agriculteurs, qui souvent ne voient pas leur maïs dans ces catégories, que les frontières entre les unes et les autres s'effacent, créant ainsi constamment des populations de plus en plus adaptées à leur besoin. Le décalage entre certaines idées reçues sur l'agriculture paysanne traditionnelle et la multitude d'agroécologies en actions au Mexique nous oblige à prendre en compte, dans les approches de la conservation, une réalité socio-économique et écologique plus complexe et problématique. 
Ce recueil vise à mettre en perspective des expériences et des analyses très variées de chercheurs issus de différentes disciplines, d'agriculteurs, d'associations. Les regards croisés entre trajectoires épistémiques, normatives et techniques opérées par ces différents groupes, constituent une occasion de remettre en relation le sujet de la biodiversité avec les identités sociales, scientifiques et politiques. Pendant les journées organisées avec le soutien du MNHN, notre but a été de faire émerger les intentions et les points de vue de chacun de ces acteurs en analysant les pratiques, les savoirs et les visions sur la biodiversité cultivée déployés dans chaque contexte.

Nous espérons que ce recueil de textes pourra mettre en évidence l'existence de plusieurs sources de la diversité cultivée. Chaque article propose des analyses sur les facteurs expliquant l'existence des variétés hétérogènes de maïs dans les parcelles des agriculteurs. Nous verrons les éléments fondateurs de la diversité des maïs mexicains basés sur le travail de plus de deux millions d'agriculteurs qui utilisent des semences issues de leur propre sélection et cultivant plus de la moitié des huit millions d'hectares consacrés annuellement à la culture du maïs (Lazos \& Chauvet 2011, Perales 2017, Bellon et al. 2018). Nous verrons aussi, en France, dans une situation tout à fait différente et au sein des réseaux associatifs comme celui d'AgroBio Périgord, que les agriculteurs ont mis en place un système également générateur de diversité.

11 L'importance du maïs dans l'agriculture paysanne au Mexique est d'abord présentée par Rafael Ortega Paczka, agronome et représentant de la Red Patrimonio Biocultural de México (Réseau Patrimoine bioculturel du Mexique). Il analyse en particulier la trajectoire historique et technique de la sélection du maïs au Mexique au prisme des différentes épistémologies, où savoirs scientifiques et savoirs paysans se mêlent. Son expérience montre le rôle crucial des agriculteurs dans l'effort d'amélioration du maïs au Mexique encourageant les approches participatives. Il prend comme modèle la «Selección Masal Visual Estratificada (SMVE) » considérée comme mettant en dialogue les savoirs écologiques des agriculteurs avec ceux des sélectionneurs.

Le numéro se poursuit avec différentes analyses issues du Proyecto Global de Maíces nativos ${ }^{5}$ (Projet global des maïs indigènes), de la Comisión nacional para el conocimiento $y$ uso de la biodiversidad (CONABIO) ${ }^{6}$, de 2006 à 2010, probablement le projet le plus exhaustif sur la distribution des maïs indigènes au Mexique jamais mis en place. L'objectif du projet était de déterminer la distribution géographique du maïs natif afin d'appréhender l'ensemble du territoire mexicain comme centre d'origine et de diversification du maïs. Chargées d'interpréter sous l'angle des sciences sociales les résultats de ce projet, Elena Lazos Chavero et Michelle Chauvet, dans le cadre d'une vaste réflexion socio-économique, analysent la diversité des mosaïques agricoles dans le nord, le centre et le sud du pays. Leur contribution est l'occasion de revenir sur différentes questions autour du maïs comme patrimoine bio-culturel. Les auteures soulignent les risques de considérer les maïs uniquement comme un capital naturel. Cecilio Mota, impliqué dans la mise en marche du « Projet Global », fournit une analyse particulièrement pertinente du travail de collecte et de systématisation des données. Il nous fait découvrir des détails surprenants de la richesse variétale du maïs et retrace également les usages de ce produit dans les pratiques alimentaires et les valeurs symboliques associées à cette diversité. Toujours en utilisant les données issues du «Projet Global » sur le maïs natif, mais pas seulement, Hugo Perales met en lumière les facteurs qui structurent le paysage variétal du maïs. Il interprète le cas du maïs au Mexique comme un exemple d'amélioration évolutive d'une plante (sélection naturelle 
et sélection menée par les agriculteurs sur une base génétique large) capable de répondre à des demandes spécifiques dans le cadre de la recherche d'une sécurité alimentaire. Son argument est construit autour de l'idée d'une conservation de facto du maïs qui serait encore très étendue et dynamique au Mexique. Il démontre ainsi comment l'usage des gènes par les sélectionneurs, ne saurait être considéré comme l'objectif principal de la conservation in situ.

En croisant différentes pistes qui guident la sociologie de l'action publique, Jean Foyer et Marianna Fenzi traitent de la problématique de la conservation en étudiant les politiques des institutions à travers la mobilisation des différents types de savoirs mis en œuvre dans le Programa de Conservación de Maíz Criollo (PROMAC). Ils montrent comment ces connaissances sont mobilisées ou au contraire marginalisées dans la perspective de rendre un objet (la conservation du maïs) gouvernable. Ils présentent les forces déterminantes dans la réalisation du programme, depuis son objectif défini au niveau des institutions nationales jusqu'à sa mise en œuvre au niveau local.

Le rôle de la culture dans les paysages agricoles est traité dans le texte de Quatzalcoatl Orozco-Ramírez et Stephen Brush, qui exposent en particulier l'influence de la langue locale dans la gestion de la diversité du maïs. Dans l'Oaxaca, les Mixtèques et les Chatines partagent un même écosystème mais se distinguent par des particularités culturelles, dont la langue. Ces particularités constituent des lignes de démarcation entre ces deux peuples, de telle sorte par exemple que, bien qu'ils soient voisins, ils ne partagent pas les mêmes lots de semences. Les noms donnés aux variétés locales enserrent les savoirs et pratiques qui sont transmis dans chaque langue, dont les subtilités peuvent difficilement être traduites. Ce cas d'étude met en évidence les forces constitutives de la diversité bi-culturelle dans cette région et montre comment celles-ci peuvent influencer les dynamiques d'évolution de cette céréale.

À partir de cette perspective qui s'intéresse aux aspects culturels intervenant dans la conservation et l'évolution de la diversité cultivée sur le terrain, l'article d'Alvaro Salgado porte son analyse sur les caractères du maïs autres que le rendement, et qui sont valorisés par les communautés paysannes et autochtones, et sur les effets résultant de ces choix. Dans son texte, Alvaro Salgado, agronome et représentant du Centro Nacional de Ayuda a las Misiones Indigenas (CENAMI) et de la Red en defensa del maíz, introduit des réflexions sur les systèmes culturels qui encadrent l'agriculture conventionnelle et paysanne, ainsi que sur les objectifs qui guident les formes $d$ 'exploitation dans chaque cas. Il montre que les différentes pratiques de sélection et de conservation produisent des résultats différents sur les populations de maïs, mais aussi sur la reproduction culturelle et l'autonomie des communautés rurales. Pour sa part, le texte de Pánfilo Hernández Ortíz, agriculteur et représentant de l'association civile Groupe Vicente Guerrero, porte sur l'expérience de défense et de protection des semences dans la région de Tlaxcala. Il retrace l'évolution des stratégies de lutte engagées au fil du temps et les acquis de ce mouvement. Il montre comment la lutte contre le maïs transgénique a non seulement abouti à la création d'une loi pour bannir son utilisation dans le département de Tlaxcala ${ }^{7}$, mais a aussi encouragé la revalorisation de 1 'agriculture paysanne et dynamisé l'échange de semences de ferme dans la région.

La dernière contribution est celle de l'association AgroBio Périgord, qui a hébergé avec le Muséum national d'Histoire naturelle, les journées d'échanges qui ont rendu possible ce numéro. Elle expose la trajectoire historique et technique d'un programme de mise en réseau des savoirs et des pratiques sur le maïs en France. Dans un contexte variétal 
homogénéisé, comme dans le cas européen, l'adoption de variétés hétérogènes a été une contribution importante au développement de systèmes agricoles diversifiés. La nécessité d'avoir sa propre semence répond ainsi au souci des agriculteurs de s'investir dans des modèles plus autonomes et façonnés par des valeurs qui sont le résultat de nouvelles formes de socialisation de la biodiversité cultivée en Europe (Fenzi \& Couix 2021). En dépit d'un contexte différent de celui du Mexique, ces agriculteurs partagent des luttes en vue de valoriser la diversité cultivée.

17 Nous avons jugé utile de compléter ce dossier sur la gestion de la biodiversité du maïs au Mexique, par deux documents liés aux activités du laboratoire d'Éco-anthropologie du Muséum national d'Histoire naturelle. Au cours des dix dernières années, les chercheurs et étudiants de ce laboratoire ont réalisé des études sur le terrain au Mexique, qui ont généré des collectes d'objets. Beaucoup de ceux-ci concernent le maïs et sa place dans l'agriculture, l'alimentation et la vie quotidienne mexicaine. Nous proposons en annexe de ce dossier, résultant des rencontres de 2014, un catalogue de 72 objets liés au maïs dans l'agriculture. Une sélection de ces objets avait été présentée dans une exposition réalisée en 2019 avec l'Institut culturel du Mexique à Paris ; nous la décrivons également dans ce numéro de la Revue d'Ethnoécologie.

Pour conclure, ce numéro voudrait souligner l'intérêt, comme dans tout débat sur la biodiversité, de reconnaitre la place centrale de l'agriculture paysanne, centrale au Mexique tant en termes de démographie, de production que de surface cultivée. Si la plupart des recherches dans ce domaine ont mis l'accent sur les conséquences $d u$ modèle agro-industriel et sur l'érosion de la biodiversité cultivée, nous nous attachons dans ce numéro à montrer l'importance de la diversité des maïs et de leur évolution, qui semblent relever d'une grande résilience même dans un contexte institutionnel qui la néglige quand il ne l'affecte pas directement. Si les agriculteurs mexicains opèrent de facto la conservation des maïs dans leurs champs, le renouvellement des stratégies de conservation sur une base interdisciplinaire et prenant en compte une diversité d'acteurs semble indispensable pour soutenir leurs pratiques. Ce numéro a le mérite de nourrir la réflexion sur la culture du maïs au Mexique pour qu'elle soit enfin considérée non pas comme un espace résiduel à utiliser comme "réservoir des gènes", mais comme un espace social et écologique encore pleinement actif et crucial pour le futur agricole de ce pays.

Les personnes qui ont contribué à ce numéro sont nombreuses et nous tenons en particulier à remercier les auteurs qui ont participé, avec ténacité, à toutes les phases de ce projet. Nous souhaitons remercier l'Action Thématique du Muséum (ATM) Savoirs naturalistes, expertise et politiques de la biodiversité, la Red Patrimonio Biocultural de México, l'association AgroBio Périgord et le Future Food Initiative qui ont soutenu les rencontres et la réalisation de ce numéro. Nous voudrions également exprimer notre reconnaissance envers l'équipe de la Revue d'Ethnoécologie ainsi qu'aux traducteurs qui ont rendu possible la publication de ces travaux: María Auxiliadora Alcivar, Françoise Aubaile, Meriene Betancourt, Mathilde Bognon, Jacqueline Ferreras, Stephanie Hamard, Catherine Hoare, Vanessa Lopez, Sebastien Plutniak, Irene Pochetti et Paul Reeve. Enfin, nous exprimons notre gratitude à toute l'équipe de la revue Etnobiología et en particulier à Eduardo Corona, Arturo Argueta et José Blancas pour avoir encouragé les échanges qui ont permis la présente publication. 


\section{BIBLIOGRAPHIE}

Aguirre Salcedo C. \& Ceccon E. 2020 - Socioecological benefits of a community-based restoration of traditional home gardens in Guerrero, Mexico. Etnobiología 18 (3) : 3-23.

Bahuchet S., Grenand F., Grenand P. \& Maret P. de 2000 - Les Peuples des forêts tropicales aujourd'hui. Vol. I, Forêts des tropiques, forêts anthropiques. Bruxelles, Commission européenne APFT., $132 \mathrm{p}$.

Bellon M.R., Mastretta-Y. A., Ponce-M. A., Ortiz-S. D., Oliveros-G. O., Perales H., Acevedo F. \& Sarukhán J. 2018 - Evolutionary and food supply implications of ongoing maize domestication by Mexican campesinos. Proceedings of the Royal Society B., 285 :20181049. doi: 10.1098/rspb.2018.1049

Bennett E. 1968 - Technical conference on the exploration, utilization and conservation of plant genetic resources. Rome, FAO. (Record of the FAO/IBP).

Bretting P.K. \& Duvick D.N. 1997 - Dynamic conservation of plant genetic resources. Advances in Agronomy $61: 1-51$.

Brush S.B. 1999 - Genes in the Field. CRC Press.

Cunha Ávila J.V. da, Anderson Santos de Mello M.E.B., Trevisan R., Fiaschi P. \& Hanazaki N. 2017 Agrobiodiversity and in situ conservation in quilombola home gardens with different intensities of urbanization. Acta Botanica Brasilica 31 (1) : 1-10. doi : 10.1590/0102-33062016abb0299

Dumez R. 2010 - Le feu, savoirs et pratiques en Cévennes. Versailles, Quæ, 245 p. (Indisciplines).

Dyer G.A., López-Feldman A., Yúnez-Naude A. \& Taylor J.E. 2014 - Genetic erosion in maize’s center of origin. Proceedings of the National Academy of Sciences $111: 14094-14099$.

FAO (2019). J. Bélanger \& D. Pilling (eds.). The State of the World's Biodiversity for Food and Agriculture, FAO Commission on Genetic Resources for Food and Agriculture Assessments. Rome. 572 pp. (http://www.fao.org/3/CA3129EN/CA3129EN.pdf)

Fenzi M. \& Bonneuil C. 2016 - From "genetic resources" to "ecosystems services": a century of science and global policies for crop diversity conservation. Culture, Agriculture, Food and Environment 38 (2) : 72-83.

Fenzi M. \& Couix N. 2021 - Growing maize landraces in industrialized countries: from the search for seeds to the emergence of new practices and values. International Journal of Agricultural Sustainability : 1-19.

Iermanó M.J., Paleologos M.F. \& Sarandón S. 2020 - Biodiversidad funcional: comprensión y evaluación para el manejo agroecológico. In : Sarandón S. (Ed.) Biodiversidad, agroecología y agricultura sustentable : 268-293. Libros de Cátedra. La Plata: Edulp.

Lazos E. \& Chauvet M. 2011 - Análisis del contexto social y biocultural de las colectas de maíces nativos en México. Proyecto Global de Maíces, Informe de Gestión, CONABIO.

Nicholls C.I., Altieri M.A., Kobayashi M., Tamura N., McGreevy S. \& Hitaka K. 2020 - Assessing the Agroecological Status of a Farm: A Principle-Based Assessment Tool for Farmers. Agro Sur 48 (2) : 29-41. doi :10.4206/agrosur.202 0.v48n2-04

Perales H.R. 2017 - Propuesta para formular un marco conceptual para las 947 actividades de conservación de las variedades nativas de maíz. Informe 948 Final del Proyecto NM001, CONABIO, Mexico. 
Perales H. \& Golicher D. 2014 - Mapping the Diversity of Maize Races in Mexico. PLOS ONE 9.

Rodríguez S. del Amo. 2011 - El Cambio de Modelo de Desarrollo Necesario en un País Pluricultural. Algunos Aspectos que se Deben Tomar en Cuenta". Etnobiología 9 (1) : 60-76.

Roué M. 2006 (Ed.) - Diversité culturelle et biodiversité. Revue Internationale des sciences sociales, $187: 187 \mathrm{p}$.

Sadras V. 2018 - Reductionism, over-simplification and plain lack of rigour can misguide R\&D investment. In : Adelaide, 26-29 November 2018, Making science useful to agriculture. University of Adelaide.

Swart Jac A.A., Zevenberg J., Ho P., Cortina J., Reed M., Derak M., Vella S., Heng Zhao \& Van der Windt H.J. 2018 - Involving Society in Restoration and Conservation. Restoration Ecology 26 (S1) : S3-6. doi : 10.1111/rec.12709

Virtanen P.K. 2019 - Ancestors' times and protection of Amazonian Indigenous biocultural heritage. AlterNative: An International Journal of Indigenous Peoples 15 (4) : 330-39. doi :

$10.1177 / 1177180119893132$

\section{NOTES}

1. La Red Patrimonio Biocultural de México est un réseau d'institutions et de chercheurs fondé en 2011 grâce au soutien du CONACyT ( ), qui a pour but d'animer les études sur la diversité biologique et culturelle du pays, ainsi que sur les différents acteurs qui la rendent possible sur le terrain comme au niveau institutionnel, voir : https://patrimoniobiocultural.com

2. Préambule, Convention sur la diversité biologique. http://www.cbd.int/doc/legal/cbd-fr.pdf

3. United Nations Declaration on the Rights of Peasants and Other People Working in Rural Areas A/HRC/ RES/39/12, (Article 19; 1d, 5,6). https://www.fao.org/family-farming/detail/fr/c/1197483/

4. Par exemple le CIMMYT présente certaines de ces activités comme relevant d'une genetic library donnant accès à une platform for the utilization of genetic resources : "The CIMMYT germplasm bank is the lifeblood of many Seeds of Discovery (SeeD) activities, preserving the genetic diversity that is necessary to develop improved maize ». https://seedsofdiscovery.org/

5. https://www.biodiversidad.gob.mx/media/1/genes/files/InformedeGestion_V1.pdf

6. La Comisión Nacional para el Conocimiento y Uso de la Biodiversidad (CONABIO) est une agence gouvernementale créée en 1992, qui a comme mission l'étude, la conservation et l'utilisation soutenable de la diversité biologique, voir : https://www.gob.mx/conabio

7. Ley de Fomento y Protección al maíz como patrimonio originario, en diversificación constante y alimentario, para el estado de Tlaxcala, publiée dans le Periódico Oficial de l'État de Tlaxcala le 18 janvier 2011. http://periodico.tlaxcala.gob.mx/indices/2Ex18012011.pdf 


\section{AUTEURS}

\section{MARIANNA FENZI}

Centre Alexandre-Koyré (EHESS/CNRS/MNHN), Campus Condorcet, Aubervilliers, France Laboratoire d'Histoire des Sciences et des Techniques, Ecole Polytechnique Fédérale de Lausanne, Lausanne, Suisse

\section{MARIA GABRIELA ZURITA-BENAVIDES}

Grupo de Ecosistemas Tropicales y Cambio Global - Universidad Regional Amazónica Ikiam JORGE QUETZAL ARGUETA PRADO

Postdoctoant Programa Interinstitucional de Especialidad en Soberanías Alimentarias y Gestión de Incidencia Local Estratégica - CIATEJ-CONACYT - México 